\title{
Risk Factors for
}

\section{Healthcare-Associated Infections Caused by Cefepime-Resistant Pseudomonas Aeruginosa}

\author{
Marko M. Folić',2, Zorana M. Djordjevićs ${ }^{3}$, Dragan R. Milovanović1,2, \\ Dejana T. Ružić Zečević1,2, Nikola V. Rosić ${ }^{1}$, Slobodan M. Janković1,2 \\ ${ }^{1}$ Clinical Centre Kragujevac, Clinical Pharmacology Department, Kragujevac, Serbia \\ ${ }^{2}$ University of Kragujevac, Faculty of Medical Science, Kragujevac, Serbia \\ ${ }^{3}$ Clinical Centre Kragujevac, Department of Hospital Infections Control, Kragujevac, \\ Serbia
}

\section{SUMMARY}

Introduction: The increasing resistance of Pseudomonas aeruginosa against cefepime requires a better understanding of hospital epidemiology in order to retain this drug as a therapeutic option.

Aim: The aim of this study was to identify risk factors associated with healthcareassociated infections (HAls) caused by cefepime-resistant $P$. aeruginosa (CRPA), both in all patients with HAls and in the subgroups of patients who died or who acquired infection with carbapenem-resistant $P$. aeruginosa.

Methods: The academic study was designed as a combination of three nested case/ control studies within the cohort of patients with HAls caused by $P$. aeruginosa regardless of localization, prospectively followed from January 2009 to December 2017 in a tertiary care hospital. The influence of risk factors on specific outcomes was estimated by adjusted odds ratios calculated from multivariate logistic regressions.

Results: Our study showed that there are independent risk factors for CRPA infections: the existence of another HAI $(p=0.001)$, hospitalization in ICU longer than one month $(p=0.007)$, a longer stay in hospital prior to infection $(p=0.004)$, longer use of mechanical ventilation $(p=0.036)$, administration of antibiotics before infection $(p<0.001)$ and previous use of fluoroquinolones $(p=0.030)$, while traumatic injury $(p=0.020)$, the existence of another HAI $(p=0.001)$ and long-term use of antibiotics before HAI $(p=0.034)$ were related to mortality. Further, independent risk factors for HAls caused by carbapenem-resistant CRPA are the existence of another HAI $(p=0.011)$, transfer from another facility $(p=0.023)$, more days spent in hospital $(p=0.006)$ and ICU $(p=0.004)$, and administration of antibiotics before infection $(\mathrm{p}<0.001)$.

Conclusion: Risk factors for HAls with CRPA are primarily linked to pre-infection use of systemic antibiotics, which is a common pathway for selection of multi, extensive or pan-resistant strains of $P$. aeruginosa that invade tissues and cause infection.

Keywords: Pseudomonas aeruginosa, cefepime, antibiotics resistance 


\section{INTRODUCTION}

In recent years, healthcare-associated infections (HAIs) caused by the bacterium Pseudomonas aeruginosa have attracted considerable interest in the medical literature, primarily due to the growing incidence of infection and mortality reported all over the world [ 1 , $2,3]$. This in turn has led to increased use of resources and consequent increases in costs in many hospitals [4]. Furthermore, this pathogen is capable of acquiring resistance to many antibiotics rapidly, combining different available mechanisms, thus rendering even our most potent agents useless. Sometimes resistance develops during the course of therapy in a single patient [5]. Timely choice of adequate antibiotic therapy is critical, especially with serious infections, since the inappropriate selection of antibiotics has been associated with adverse outcomes in the past [6].

Cefepime is a fourth-generation cephalosporin antibiotic with good activity against important pathogens including $P$. aeruginosa, and it was designated as a front-line agent to treat moderate to severe HAIs, including pneumonia and infections of the urinary tract and skin [7]. However, the growing frequency of cefepime-resistant $P$. aeruginosa (CRPA) as a cause of these infections frequently complicates treatment and compromises its results. Research to date has aimed at identifying some of the risk factors for the occurrence of HAIs caused by this pathogen such as: prior use of certain antibiotics [8]. However, there is still a gap in our clinical knowledge and inconsistencies regarding the risk factors connected to the deaths of the patients with such infections.

Deeper understanding of the hospital epidemiology of HAIs caused by P. aeruginosa would contribute to the retention of cefepime as a therapeutic option for a longer period of time [9]. Identifying subgroups of patients who are at increased risk for acquisition of these infections in the hospital setting will assist in providing timely and appropriate treatment. Additional knowledge is needed about the prevention of HAIs caused by CRPA and rational choice of antimicrobial therapy particularly since we know that, in the foreseeable future, we cannot expect a new antibiotic aimed at $P$. aeruginosa.
AIM

The aim of our study was to identify risk factors associated with HAIs caused by CRPA, not only in the whole population of patients with HAIs, but also in the subgroups of patients who died in a hospital or acquired an HAI with carbapenem-resistant $P$. aeruginosa.

\section{METHODS}

The academic (noncommercial) IV phase nonintervention prospective study was designed as three nested case/control studies within the cohort of patients with HAIs, prospectively followed from January 2009 to December 2017 in a tertiary care hospital, the "Clinical Centre Kragujevac" (CCKg), placed in the town Kragujevac, Serbia. The study was reviewed and approved by the Ethics Committee of the CCKg (No: 01/43). Written informed consent was obtained from each patient before the initiation of any procedure related to this research. The CCkg has about 1200 beds and cares for 50,000 inpatients per year.

The inclusion criteria for the study cohort were age over 18 and acquired HAI with $P$. aeruginosa, regardless of localization. Only the first isolate from the same infection site during hospitalization was taken into account for the purposes of this study. The exclusion criteria were the isolation of $P$. aeruginosa within the first 48 hours after admission to hospital and colonization of the patient with this pathogen.

Diagnosis of HAI and determination of the infection site were made according to the criteria of the Center for Disease Control and Prevention (CDC), Atlanta, United States [10], which require that the patient does not have an infection on admission to hospital and is not in the state of incubation. Surveillance of HAIs was performed prospectively and the data obtained from the patient's history, physical examination, a search of the patients' files, microbiological, biochemical, and hematological analyses. They were entered into a specially designed questionnaire. Each patient was evaluated by the investigators with the aim of excluding patients with $P$. aeruginosa colonization.

The following potential risk factors were taken into account: (a) intrinsic factors: gender, age, comorbidities or conditions (diabetes mellitus, asthma, heart failure, traumatic 
injury based on International Classification of Diseases Ten Revision Codes and existence of other HAI, etc.), (b) factors related to healthcare: dates of admission and discharge from hospital and intensive care units (ICU), transfer from another facility (i.e., the same or another hospital, long-term care facility, rehabilitation center), administration of medical devices (urinary catheter, mechanical ventilation, etc.), the date the HAI was acquired, and (c) administration of antibiotics: name, starting and ending date of antibiotic administration. For the purposes of this study, previous use of antibiotics was defined as any antimicrobial agent administered systemically for at least 24 hours during the 14-day period before the isolation of $P$. aeruginosa.

Isolation and identification of causative agents of HAIs were conducted in the Microbiology Laboratory of CCKg using conventional biochemical methods [11], while interpretation of the results followed the guidelines issued by the Clinical and Laboratory Standards Institute [12]. The $P$. aeruginos $a$ isolates were tested for resistance using standard methods to the following antibiotics (breakpoints given in parentheses): imipenem $(10 \mu \mathrm{g} / \mathrm{mL})$, meropenem $(10 \mu \mathrm{g} / \mathrm{mL})$, cefotaxime $(30 \mu \mathrm{g} / \mathrm{mL})$, ceftriaxone $(30 \mu \mathrm{g} /$ $\mathrm{mL})$, ceftazidime $(30 \mu \mathrm{g} / \mathrm{mL})$, cefepime $(30 \mu \mathrm{g} /$ $\mathrm{mL})$, gentamicin $(10 \mu \mathrm{g} / \mathrm{mL})$, amikacin $(30 \mu \mathrm{g} /$ $\mathrm{mL})$, ciprofloxacin $(5 \mu \mathrm{g} / \mathrm{mL})$, tigecycline $(15$ $\mu \mathrm{g} / \mathrm{mL})$ and piperacillin-tazobactam $(110 \mu \mathrm{g} /$ $\mathrm{mL}$ ). The tested isolates were classified as susceptible or resistant (including intermediate) strains. Multi-resistance was defined as acquired non-susceptibility to at least one agent from three or more different antibiotic groups, and pan-resistance as non-susceptibility to all antimicrobial categories [13]. For the purpose of univariate and multivariate statistical analysis, antimicrobial drugs were categorized by agents or classes. The class of antibiotics is a group of different drugs with similar chemical and pharmacological properties, and their effect is expressed by the same or related bacteria.

Based on the susceptibility of isolated $P$. aeruginosa, which caused the HAI, to cefepime, the patients were at first classified in one of two groups: cases, i.e. patients with HAI caused by CRPA, and controls, i.e. patients with HAI caused by cefepime-sensitive $P$. aeruginosa (CSPA). Second, the patients who died within 30 days following infection with $P$. aeruginosa were classified into two subgroups: the cases were the patients with HAI caused by CRPA, and the controls were the patients with HAI caused by CSPA. The third nested case/ control study was carried out on the patients who had acquired HAI caused by P. aeruginosa resistant to carbapenems: the cases were the patients whose isolates were also resistant to cefepime (CRPA-CR), and the controls were those infected with cefepime-sensitive strains of carbapenem-resistant $P$. aeruginosa (CSPA$\mathrm{CR})$.

The data were processed using the statistical software SPSS for Windows, version 18 (Chicago: SPSS Inc.). Primary data were analyzed by descriptive statistics using measures of central tendency, dispersion, and relative numbers. Incidence density was calculated as the number of isolates per 1,000 patient-days in that year. The trend over time of the incidence density of the isolates was determined by linear regression with the yearly data. After the data were tested for normality of distribution, differences among the groups with respect to continuous variables were tested with Student's $t$ test, while differences in categorical variables were examined using the Chi-square or Fisher's test. Association of potential risk factors with outcomes was tested by univariate and multivariate logistic regression and expressed as crude or adjusted odds ratios. Differences were considered statistically significant if the probability of the null hypothesis was below 0.05 .

\section{RESULTS}

According to the previously adopted diagnostic criteria, 847 hospitalized patients acquired a total of 869 HAIs caused by $P$. aeruginosa. The average age of the patients was $62.03 \pm 15.6$ (range: 18-93 years). Men were more frequent $(\mathrm{n}=582 ; 66.97 \%)$. Representing $15.56 \%$ of all HAIs registered in this hospital. These infections were second only to HAIs caused by the bacterium Acinetobacter spp.

The highest number of HAIs caused by $P$. aeruginosa and incidence densities during the study period was recorded in 2010 ( $\mathrm{n}=143 ; 0.57 / 1,000$ patient-days), and the smallest number and the lowest incidence densities in 2014 ( $n=57 ; 0.23 / 1,000$ patient-days). The analysis of the incidence densities shows an increasing trend for the observed period, but without statistical significance $(y=0.002 x$ - 
Table 1. Risk factor for healthcare-associated infections with cefepime-resistant Pseudomonas aeruginosa

*Shown when significant; Results are presented as \pm SD or $\mathrm{n}(\%)$;

HAls: healthcare-associated infections;

aOR: adjusted odds ratio;

$\mathrm{Cl}$ : confidence interval;

CRPA: cefepime-resistant Pseudomonas aeruginosa;

CSPA: cefepime-sensitive Pseudomonas aeruginosa; ICU: intensive care unit.

\begin{tabular}{|c|c|c|c|c|c|}
\hline \multirow[b]{2}{*}{ Variable } & \multicolumn{3}{|c|}{ Univariate analysis } & \multicolumn{2}{|c|}{ Multivariate analysis* } \\
\hline & $\begin{array}{c}\text { CRPA } \\
\mathrm{n}=598(\%)\end{array}$ & $\begin{array}{c}\text { CSPA } \\
n=271(\%)\end{array}$ & $\mathbf{p}$ & aOR $(95 \% \mathrm{Cl})$ & $\mathbf{p}$ \\
\hline Age & $61.80 \pm 15.26$ & $62.52 \pm 16.38$ & 0.529 & & \\
\hline$>65$ years of age & $292(48.8)$ & $137(50.6)$ & 0.638 & & \\
\hline Male gender & $407(68.1)$ & $169(62.4)$ & 0.100 & & \\
\hline \multicolumn{6}{|l|}{ Comorbidities } \\
\hline Diabetes mellitus & $56(9.4)$ & $28(10.3)$ & 0.655 & & \\
\hline Injury & $166(27.8)$ & $64(23.6)$ & 0.200 & & \\
\hline Existence of other HAls & $267(44.6)$ & $73(26.9)$ & $<0.001$ & $2.257(1.383-3.684)$ & 0.001 \\
\hline \multicolumn{6}{|l|}{ Hospitalization } \\
\hline $\begin{array}{l}\text { Transfer from another } \\
\text { facility }\end{array}$ & $361(60.4)$ & $112(41.3)$ & $<0.001$ & & \\
\hline Hospital (days) & $35.67 \pm 17.21$ & $30.94 \pm 13.84$ & $<0.001$ & & \\
\hline Hospital > 1 month & 345 (57.7) & $132(48.7)$ & 0.014 & & \\
\hline Stay in ICU & 466 (77.9) & $197(72.7)$ & 0.093 & & \\
\hline ICU (days) & $23.42 \pm 11.79$ & $19.97 \pm 11.07$ & $<0.001$ & & \\
\hline ICU > 1 month & $172(36.9)$ & $40(20.3)$ & $<0.001$ & $2.440(1.274-4.672)$ & 0.007 \\
\hline Length before HAI (days) & $19.94 \pm 12.18$ & $14.13 \pm 9.20$ & $<0.001$ & $1.049(1.016-1.084)$ & 0.004 \\
\hline \multicolumn{6}{|l|}{ Medical devices } \\
\hline Urinary catheter & $563(94.1)$ & $253(93.4)$ & 0.652 & & \\
\hline Urinary catheter (days) & $20.66 \pm 13.12$ & $18.19 \pm 10.72$ & 0.009 & & \\
\hline Mechanical ventilation & $299(50.0)$ & $122(45.0)$ & 0.173 & & \\
\hline $\begin{array}{l}\text { Mechanical ventilation } \\
\text { (days) }\end{array}$ & $13.78 \pm 11.65$ & $10.89 \pm 8.80$ & 0.014 & $1.035(1.002-1.070)$ & 0.036 \\
\hline \multicolumn{6}{|l|}{ Antibiotics before HAls } \\
\hline Use of antibiotics & $577(96.5)$ & $246(90.8)$ & $<0.001$ & $1.755(1.341-2.297)$ & $<0.001$ \\
\hline No antibiotics & $2.73 \pm 1.17$ & $2.13 \pm 0.94$ & $<0.001$ & & \\
\hline Days of antibiotic therapy & $15.46 \pm 7.91$ & $11.80 \pm 6.93$ & $<0.001$ & & \\
\hline Piperacillin-tazobactam & $71(11.9)$ & $12(4.4)$ & $<0.001$ & & \\
\hline Carbapenems & $217(36.6)$ & $78(28.8)$ & 0.030 & & \\
\hline $3^{\text {rd }}$ gen. cephalosporin & $234(39.1)$ & $79(29.2)$ & 0.005 & & \\
\hline Cefepime & $26(4.3)$ & $8(3.0)$ & 0.326 & & \\
\hline Aminoglycosides & $245(41.0)$ & $89(32.8)$ & 0.022 & & \\
\hline Fluoroquinolone & $149(24.9)$ & $41(15.1)$ & 0.001 & $2.074(1.074-4.006)$ & 0.030 \\
\hline Glycopeptide & $155(25.9)$ & $52(19.2)$ & 0.031 & & \\
\hline Metronidazol & $144(24.1)$ & $50(18.5)$ & 0.065 & & \\
\hline Colistine & $23(3.8)$ & $6(2.2)$ & 2.215 & & \\
\hline Tigecycline & $26(4.3)$ & $10(3.7)$ & 0.652 & & \\
\hline
\end{tabular}

4.310; R2=0.003; $\mathrm{p}=0.875 ;$ Data not shown).

In $598(68.81 \%)$ patients the HAIs were caused by CRPA and these were our cases, while the other 271 (31.82\%) of HAIs were caused by CSPA and became our control group. The share of specific sites of HAIs was not statistically different between the groups $(\mathrm{p}>0.05)$. The dominant anatomical localizations of the HAIs in both groups were pneumonia ( $48.9 \%$ vs. $47.2 \%$, respectively) and surgical site infection $(24.9 \%$ vs. $27.7 \%$, respectively), followed by infection of the urinary tract $(19.2 \%$ vs. $18.8 \%$, respectively), bloodstream ( $2.5 \%$ vs. $3.3 \%$, respectively) and other sites ( $4.5 \%$ vs. $3.0 \%$, respectively).

The results of the univariate analyses of possible risk factors for acquiring HAIs caused by CRPA, including demographic and clinical characteristics, invasive procedures, and prior therapy are shown in Table 1 . The 


\begin{tabular}{|c|c|c|c|c|c|}
\hline \multirow[b]{2}{*}{ Variable } & \multicolumn{3}{|c|}{ Univariate analysis } & \multicolumn{2}{|c|}{ Multivariate analysis* } \\
\hline & $\begin{array}{c}\text { CRPA } \\
\mathrm{n}=217(\%)\end{array}$ & $\begin{array}{c}\text { CSPA } \\
n=69(\%)\end{array}$ & $\mathbf{p}$ & aOR $(95 \% \mathrm{Cl})$ & $\mathbf{p}$ \\
\hline Age & $64.96 \pm 14.65$ & $67.20 \pm 14.66$ & 0.269 & & \\
\hline$>65$ years of age & $122(56.2)$ & $43(62.3)$ & 0.372 & & \\
\hline Male gender & $144(66.4)$ & $45(65.2)$ & 0.861 & & \\
\hline \multicolumn{6}{|l|}{ Comorbidities } \\
\hline Diabetes mellitus & $28(12.9)$ & $7(10.1)$ & 0.543 & & \\
\hline Injury & $48(22.1)$ & $5(7.2)$ & 0.006 & $\begin{array}{c}12.639(1.483- \\
107.687)\end{array}$ & 0.020 \\
\hline Existence of other HAls & $111(51.2)$ & $18(26.1)$ & $<0.001$ & $\begin{array}{c}4.599(1.832- \\
11.541)\end{array}$ & 0.001 \\
\hline \multicolumn{6}{|l|}{ Hospitalization } \\
\hline $\begin{array}{l}\text { Transfer from another } \\
\text { facility }\end{array}$ & $130(59.9)$ & $35(50.7)$ & 0.179 & & \\
\hline Hospital (days) & $34.44 \pm 18.29$ & $30.57 \pm 16.34$ & 0.117 & & \\
\hline Hospital > 1 month & $113(52.1)$ & $34(49.3)$ & 0.685 & & \\
\hline Stay in ICU & $191(88.0)$ & $63(91.3)$ & 0.451 & & \\
\hline ICU (days) & $24.97 \pm 11.63$ & $21.46 \pm 11.75$ & 0.039 & & \\
\hline ICU > 1 month & $72(37.7)$ & $15(23.8)$ & 0.044 & & \\
\hline Length before HAI (days) & $19.65 \pm 11.55$ & $15.51 \pm 10.24$ & 0.008 & & \\
\hline \multicolumn{6}{|l|}{ Medical devices } \\
\hline Urinary catheter & $211(97.2)$ & $69(100.0)$ & 0.163 & & \\
\hline Urinary catheter (days) & $25.21 \pm 15.71$ & $22.26 \pm 14.29$ & 0.168 & & \\
\hline Mechanical ventilation & $143(65.9)$ & $43(62.3)$ & 0.587 & & \\
\hline $\begin{array}{l}\text { Mechanical ventilation } \\
\text { (days) }\end{array}$ & $17.48 \pm 13.76$ & $12.12 \pm 10.01$ & 0.019 & & \\
\hline \multicolumn{6}{|l|}{ Antibiotics before HAls } \\
\hline Use of antibiotics & $214(98.6)$ & $67(97.1)$ & 0.403 & & \\
\hline No antibiotics & $2.85 \pm 1.16$ & $2.19 \pm 0.93$ & $<0.001$ & & \\
\hline Days of antibiotic therapy & $15.72 \pm 7.58$ & $11.72 \pm 7.04$ & $<0.001$ & $1.118(1.008-1.238)$ & 0.034 \\
\hline Piperacillin-tazobactam & $30(13.8)$ & $2(2.9)$ & 0.012 & & \\
\hline Carbapenems & $96(44.2)$ & $27(39.1)$ & 0.455 & & \\
\hline $3^{\text {rd }}$ gen. cephalosporin & $92(42.4)$ & $23(33.3)$ & 0.181 & & \\
\hline Cefepime & $6(2.8)$ & $3(4.3)$ & 0.512 & & \\
\hline Aminoglycosides & $79(36.4)$ & $20(29.0)$ & 0.259 & & \\
\hline Fluoroquinolone & $54(24.9)$ & $13(18.8)$ & 0.302 & & \\
\hline Glycopeptide & $65(30.0)$ & $16(23.3)$ & 0.277 & & \\
\hline Metronidazol & $66(30.4)$ & $14(20.3)$ & 0.103 & & \\
\hline Colistine & $11(5.1)$ & $2(2.9)$ & 0.451 & & \\
\hline
\end{tabular}

Table 2. Risk factors for healthcare-associated infections caused by cefepime-resistant Pseudomonas aeruginosa in patients who died

*Shown when significant;

Results are presented as $\pm \mathrm{SD}$ or n (\%);

HAls: healthcare-associated infections;

aOR: adjusted odds ratio;

$\mathrm{Cl}$ : confidence interval;

CRPA: cefepime-resistant Pseudomonas aeruginosa;

CSPA: cefepime-sensitive Pseudomonas aeruginosa; ICU: intensive care unit. following risk factors were significantly associated with CRPA infections: the existence of another HAI $(\chi 2=24.562 ; \mathrm{p}<0.001)$, transfer from another facility $(\chi 2=27.225 ; \mathrm{p}<0.001)$, more days spent in hospital and ICU $(\mathrm{t}=$ 3.982; $\mathrm{p}<0.001$ and $\mathrm{t}=3.508 ; \mathrm{p}<0.001$ respectively), especially when it was more than a month $\left(\chi 2=6.079 ; \mathrm{p}=0.014\right.$ and $\chi^{2}=17.553$; $\mathrm{p}<0.001$ respectively), longer stay in hospi- tal until infection $(\mathrm{t}=7.003 ; \mathrm{p}<0.001)$, longer period of urinary catheter in situ $(\mathrm{t}=2.624$; $\mathrm{p}=0,009)$ or longer dependence on mechanical ventilation $(\mathrm{t}=2.467 ; \mathrm{p}=0.014)$, administration of antibiotics before HAI $(\chi 2=12.143$; $\mathrm{p}<0.001)$, large number of prescribed antibiotics $(t=7.048 ; \mathrm{p}<0.001)$ and their longer use $(\mathrm{t}=6.303 ; \mathrm{p}<0.001)$, previous administration of piperacilin-tazobactam $(\chi 2=11.965 ; \mathrm{p}<0.001)$, 
carbapenems $(\chi 2=4.685 ; \mathrm{p}=0.030)$, third generation cephalosporins $(\chi 2=8.059 ; \mathrm{p}=0.005)$, aminoglycosides $(\chi 2=5.207 ; \mathrm{p}=0.022)$, fluoroquinolones $(\chi 2=10.457 ; \mathrm{p}<0.001)$ or glycopeptides $(\chi 2=4.657 ; \mathrm{p}=0.031)$

Multivariate logistic regression identified six independent risk factors for CRPA infections: the existence of another HAI $\quad(\mathrm{aOR}=2.257 ; \quad 95 \% \quad \mathrm{CI}=1.383-3.684$; $\mathrm{p}=0.001)$, hospitalization in ICU longer than one month $(\mathrm{aOR}=2.440 ; 95 \% \mathrm{CI}=1.247-4.672$; $\mathrm{p}=0.007)$, longer stay in hospital until infection $(\mathrm{aOR}=1.049 ; \quad 95 \% \quad \mathrm{CI}=1.016-1.084$; $\mathrm{p}=0.004$ ), longer use of mechanical ventilation $\quad(\mathrm{aOR}=1.035 ; \quad 95 \% \quad \mathrm{CI}=1.002-1.070$; $\mathrm{p}=0.036$ ), administration of antibiotics before infection $(\mathrm{aOR}=1.755 ; 95 \% \mathrm{CI}=1.341-2.297$; $\mathrm{p}<0.001)$ and previous use of fluoroquinolones
Table 3. Risk factor for healthcare-associated infections caused by cefepime-resistant Pseudomonas aeruginosa in patients harboring carbapenemresistant $P$. aeruginosa.

*Shown when significant; Results are presented as \pm SD or n (\%);

HAls: hospital infections;

aOR: adjusted odds ratio;

$\mathrm{Cl}$ : confidence interval;

CRPA-CR: cefepime-resistant Pseudomonas aeruginosa in patients harboring carbapenemresistant $P$. aeruginosa;

CSPA: cefepime-sensitive Pseudomonas aeruginosa in patients harboring carbapenem-resistant $P$. aeruginosa;

ICU: intensive care unit.

\begin{tabular}{|c|c|c|c|c|c|}
\hline \multirow[b]{2}{*}{ Variable } & \multicolumn{3}{|c|}{ Univariate analysis } & \multicolumn{2}{|c|}{ Multivariate analysis* } \\
\hline & $\begin{array}{c}\text { CRPA-CR } \\
n=431(\%)\end{array}$ & $\begin{array}{l}\text { CSPA-CR } \\
n=113(\%)\end{array}$ & $\mathbf{p}$ & aOR $(95 \% \mathrm{Cl})$ & $\mathbf{p}$ \\
\hline Age & $61.37 \pm 15.37$ & $59.15 \pm 18.00$ & 0.189 & & \\
\hline$>65$ years of age & $206(47.8)$ & $46(40.7)$ & 0.179 & & \\
\hline Male gender & $298(69.1)$ & $69(61.1)$ & 0.103 & & \\
\hline \multicolumn{6}{|l|}{ Comorbidities } \\
\hline Diabetes mellitus & $40(9.3)$ & $6(5.3)$ & 0.177 & & \\
\hline Injury & $122(28.3)$ & $25(22.1)$ & 0.188 & & \\
\hline Existence of other HAls & $203(47.1)$ & $33(29.2)$ & $<0.001$ & $2.368(1.219-4.598)$ & 0.011 \\
\hline \multicolumn{6}{|l|}{ Hospitalization } \\
\hline $\begin{array}{l}\text { Transfer from another } \\
\text { facility }\end{array}$ & $266(61.7)$ & $49(43.4)$ & $<0.001$ & $2.174(1.114-4.245)$ & 0.023 \\
\hline Hospital (days) & $36.05 \pm 16.90$ & $31.98 \pm 15.92$ & 0.022 & $1.057(1.028-1.088)$ & 0.006 \\
\hline Hospital > 1 month & 254 (58.9) & $59(52.2)$ & 0.198 & & \\
\hline Stay in ICU & $348(80.7)$ & $95(84.1)$ & 0.418 & & \\
\hline ICU (days) & $23.64 \pm 11.74$ & $19.64 \pm 11.47$ & 0.003 & $1.064(1.020-1.109)$ & 0.004 \\
\hline $\mathrm{ICU}>1$ month & $131(37.6)$ & $21(22.1)$ & 0.005 & & \\
\hline Length before HAI (days) & $20.19 \pm 12.08$ & $14.49 \pm 10.14$ & $<0.001$ & & \\
\hline \multicolumn{6}{|l|}{ Medical devices } \\
\hline Urinary catheter & $405(94.0)$ & $109(96.5)$ & 0.301 & & \\
\hline Urinary catheter (days) & $21.13 \pm 13.55$ & $18.71 \pm 11.78$ & 0.091 & & \\
\hline Mechanical ventilation & $229(53.1)$ & $66(58.4)$ & 0.316 & & \\
\hline $\begin{array}{l}\text { Mechanical ventilation } \\
\text { (days) }\end{array}$ & $14.17 \pm 12.45$ & $10.88 \pm 8.84$ & 0.046 & & \\
\hline \multicolumn{6}{|l|}{ Antibiotics before HAls } \\
\hline Use of antibiotics & $421(97.7)$ & $106(93.8)$ & 0.035 & $1.810(1.299-2.522)$ & $<0.001$ \\
\hline No antibiotics & $2.77 \pm 1.14$ & $2.21 \pm 0.96$ & $<0.001$ & & \\
\hline Days of antibiotic therapy & $15.48 \pm 7.63$ & $11.85 \pm 7.48$ & $<0.001$ & & \\
\hline Piperacillin-tazobactam & $49(11.4)$ & $8(7.1)$ & 0.185 & & \\
\hline Carbapenems & $173(40.1)$ & $47(41.6)$ & 0.779 & & \\
\hline $3^{\text {rd }}$ gen. cephalosporin & $173(40.1)$ & $32(28.3)$ & 0.021 & & \\
\hline Cefepime & $19(4.4)$ & $3(2.7)$ & 0.400 & & \\
\hline Aminoglycosides & $182(42.2)$ & $34(30.1)$ & 0.019 & & \\
\hline Fluoroquinolone & $101(23.4)$ & $19(16.8)$ & 0.131 & & \\
\hline Glycopeptide & $125(29.0)$ & $26(23.0)$ & 0.205 & & \\
\hline Metronidazol & $108(25.1)$ & $16(14.2)$ & 0.014 & & \\
\hline Colistine & $19(4.4)$ & $3(2.7)$ & 0.400 & & \\
\hline Tigecycline & $23(5.3)$ & $7(6.2)$ & 0.722 & & \\
\hline
\end{tabular}


$(\mathrm{aOR}=2.074 ; 95 \% \mathrm{CI}=1.074-4.006 ; \mathrm{p}=0.030)$ (Table 1).

From the total study cohort, 274 patients, with 286 HAIs, died in the first 30 days after diagnosis of infection caused by $P$. aeruginosa. Their mean age was $65.5 \pm 14.66$ years (range: 20-93 years), and 192 of them (67.1\%) were men. Somewhat more than three-quarters of these patients contracted an HAI caused by CRPA $(\mathrm{n}=217 ; 75.9 \%)$, while the rest got CSPA ( $\mathrm{n}=69 ; 24.1 \%)$.

Results of the univariate analyses of the risk factors for HAI caused by CRPA in patients who died are shown in Table 2. The following risk factors were significantly associated with CRPA infection in patients who died: comorbidities such as traumatic injury $(\chi 2=7.671 ; \mathrm{p}=0.006)$ and the existence of another HAI $(\chi 2=13.284 ; \mathrm{p}<0.001)$, more days spent in ICU $(t=2,070 ; p=0.039)$, especially when it was more than a month $(\chi 2=4.057$; $\mathrm{p}=0.044)$ and a longer stay in the hospital until infection ( $t=2.664 ; \mathrm{p}=0.008)$, longer use of mechanical ventilation $(\mathrm{t}=2.374 ; \mathrm{p}=0.019)$, the larger number of prescribed antibiotics $(t=4.203 ; \mathrm{p}<0.001)$ and their longer use before HAI $(\mathrm{t}=3.830 ; \mathrm{p}<0.001)$, and previous administration of piperacillin-tazobactam $(\chi 2=6.290 ; \mathrm{p}=0.012)$.

Multivariate logistic regression identified three independent risk factors for HAIs caused by CRPA in patients who died: traumatic injury $(\mathrm{aOR}=12.639$; $95 \% \mathrm{CI}=1.481-$ 107.687; $\mathrm{p}=0.020)$, existence of another HAI $(\mathrm{aOR}=4.599 ; 95 \% \mathrm{CI}=1.832-11.541 ; \mathrm{p}=0.001)$ and long-term use antibiotics before HAI $(\mathrm{aOR}=1.118 ; 95 \% \mathrm{CI}=1.008-1.238 ; \mathrm{p}=0.034)$ (Table 2).

Of the total number of patients initially enrolled, 544 of them had HAI caused by $P$. aeruginosa resistant to carbapenems. There were $431(79.2 \%)$ patients who had an infection caused by CRPA-CR and 113 (20.8\%) patients with CSPA-CR infections.

Table 3 shows the results of the univariate analyses of possible risk factors for HAIs caused by CRPA in patients harboring carbapenem-resistant $P$. aeruginosa. The following risk factors were significantly associated with these infections: the existence of another HAI $(\chi 2=11.674 ; p=0.001)$, transfer from another facility $(\chi 2=12.373 ; \mathrm{p}<0.001)$, more days spent in hospital $(\mathrm{t}=2.304$; $\mathrm{p}=0.022)$ and ICU $(\mathrm{t}=2.960 ; \mathrm{p}=0.003)$, hospitalization in ICU longer than one month $(\chi 2=7.994 ; \mathrm{p}=0.005)$, longer stay in hospital until infection $(\mathrm{t}=4.611 ; \mathrm{p}<0.001)$, longer use of mechanical ventilation $(t=2.003 ; p=0.046)$, administration of antibiotics before HAI infection $(\chi 2=4.439 ; p=0.035)$, larger number of prescribed antibiotics $(\mathrm{t}=4.684 ; \mathrm{p}<0.001)$ and their longer use $(t=4.392 ; \mathrm{p}<0.001)$, and previous administration third-generation cephalosporins $(\chi 2=5.327 ; \mathrm{p}=0.021)$, aminoglycoside $(\chi 2=5.510 ; \mathrm{p}=0.019)$ or metronidazole $\left(\chi^{2}=6.043 ; \mathrm{p}=0.014\right)$.

Multivariate logistic regression identified five independent risk factors for CRPA in patients with carbapenem-resistant $P$. aeruginosa hospital infections: the existence of another HAI $(\mathrm{aOR}=2.368$; $95 \% \mathrm{CI}=2.219$ 4.598; $\mathrm{p}=0.011)$, transfer from another facility $(\mathrm{aOR}=2.174 ; 95 \% \mathrm{CI}=1.114-4.245 ; \mathrm{p}=0.023)$, more days spent in hospital $(\mathrm{aOR}=1.057$; 95\% CI $=1.028-1.088 ; \mathrm{p}=0.006)$ and ICU (aOR=1.064; 95\% CI=1.020-1.109; $\mathrm{p}=0.004)$, and administration of antibiotics before infection $(\mathrm{aOR}=1.810 ; 95 \% \quad \mathrm{CI}=1.299-2.522$; $\mathrm{p}<0.001$ ) (Table 3 ).

The isolates of $P$. aeruginosa showed a high resistance rate $(70-97 \%)$ to the following classes of antibiotics: cephalosporins, amino-

\begin{tabular}{|c|c|c|c|}
\hline Antimicrobial agent & $\begin{array}{c}\text { CRPA } \\
\text { n/N (\%) }\end{array}$ & $\begin{array}{c}\text { CSPA } \\
\text { n/N (\%) }\end{array}$ & $\mathbf{p}$ \\
\hline Imipenem & $392 / 581(67.5)$ & $87 / 264(33.0)$ & $<0.001$ \\
\hline Meropenem & $420 / 585(71.8)$ & $111 / 264(42.0)$ & $<0.001$ \\
\hline Cefotaxime & 498/501 (99.4) & $209 / 234(89.3)$ & $<0.001$ \\
\hline Ceftriaxone & $552 / 555(99.5)$ & $227 / 250(90.8)$ & $<0.001$ \\
\hline Ceftazidime & $571 / 580(98.4)$ & $172 / 249(69.1)$ & $<0.001$ \\
\hline Gentamicin & $490 / 521(94.0)$ & $198 / 239(82.8)$ & $<0.001$ \\
\hline Amikacin & $449 / 582$ (77.1) & $142 / 256(55.0)$ & $<0.001$ \\
\hline Ciprofloxacin & $522 / 567(92.1)$ & $194 / 257(75.5)$ & $<0.001$ \\
\hline Tigecyclin & $28 / 61(45.9)$ & $8 / 19(42.1)$ & 0.771 \\
\hline Piperacillin-tazobactam & 166/555 (29.9) & $34 / 232(14.7)$ & $<0.001$ \\
\hline
\end{tabular}

Table 4. Comparison of antimicrobial resistance of Pseudomonas aeruginosa to selected antibiotics

$\mathrm{n}$ : number of resistant isolates and $\mathrm{N}$ : number of isolates with available results;

CRPA-carbapenem-resistant Pseudomonas aeruginosa; CSPA-carbapenem-sensitive Pseudomonas aeruginosa 
glycosides, and ciprofloxacin. The moderate resistance rate was registered to carbapenems (57-63\%), while resistance to tigecycline and piperacillin-tazobactam was somewhat lower (45 and 57\%). Comparing the resistance rates of CRPA and CSPA isolates to antibiotics, a difference was noted for both carbapenems, cephalosporins, aminoglycosides, ciprofloxacin, and piperacillin-tazobactam $(\mathrm{p}<0.05)$, while it was similar to tigecycline (Table 4). Nearly $85 \%(\mathrm{n}=738)$ of $P$. aeruginosa isolates from both groups were multi-resistant, while about $10 \%(\mathrm{n}=84)$ of isolates were pan-resistant.

\section{DISCUSSION}

Our study showed that concomitant HAI is a significant risk factor for CRPA in all patients with HAIs caused by $P$. aeruginosa, but also for patients who died or acquired carbapenemresistant $P$. aeruginosa. Other important risk factors were a longer stay in the hospital environment (in hospital in general, in ICU, or in other departments), receiving antibiotics, especially for prolonged periods, and prescription of a fluoroquinolone, being on mechanical ventilation and having a traumatic injury as one of the reasons for hospitalization.

Concomitant HAI was already confirmed as a significant risk factor for HAIs caused by $P$. aeruginosa producing metallo$\beta$-lactamase, which is in more than $90 \%$ of cases resistant to cefepime $[14,15]$. In tertiary care hospitals, urinary tract infection acquired during hospitalization increased by 2.48 times the chances of becoming infected with $P$. aeruginosa producing metallo- $\beta$-lactamase, and therefore resistant to cefepime [16]. The mechanism of increased risk of infection with CRPA in patients with urinary tract infections is increased exposure to beta-lactam antibiotics and fluoroquinolones, which produce selective pressure on bacterial flora and promote the growth of resistant strains of $P$. aeruginosa [16].

In the hospital environment, $P$. aeruginosa is prone to become resistant to antibiotics from multiple classes, and it has previously been shown that its resistance to one class of antibiotics is associated with resistance to other classes [8]. Although it was thought that due to changes in the side chain of its molecule, cefepime does not produce stable derepression of the AmpC $\beta$-lactamase as ceftazidime does, and therefore $P$. aeruginosa is less resistant to cefepime than to ceftazidime, recent studies offered proof that cefepime may still activate AmpC beta-lactamase and lose its potency; this process of acquiring resistance is promoted by previous use of beta-lactam antibiotics and fluoroquinolones, but aminoglycosides, on the contrary, may block it and preserve susceptibility to cefepime $[8,17]$. Besides, strains of $P$. aeruginosa that are extensive producers of metallo- $\beta$-lactamases are resistant to cefepime, and they cause HAIs more often in patients that receive antibiotics for treatment of urinary tract infections and stay longer in hospital [18]. Many other studies have shown that a prolonged stay in a hospital, and especially in ICU, is a clear risk factor for acquiring multidrug-resistant, extensively drug-resistant, and pan drug-resistant $P$. aeruginos $a$ which is resistant to cefepime among other antibiotics [6]. It was calculated that the period between the $7^{\text {th }}$ and $14^{\text {th }}$ day of hospitalization had the highest HAI rate with Gram-negative bacteria including $P$. aeruginosa, probably because the prescription rate for antibiotics is highest in the first two weeks of a hospital stay [19].

Previous use of fluoroquinolones is one of the most potent drivers of HAIs with CRPA, even stronger than previous exposure to cefepime itself (odds ratio 14.7 vs. 8.5) [20]. Although the exact mechanism of the exposure to one group of antibiotics leads to resistance to other groups is still unknown, one possibility is that in organisms with multiple resistance mechanisms, such as $P$. aeruginosa, high exposure to partially efficient antibiotic results in joint induction of many different and unrelated mechanisms of resistance, because their expression could be linked. Exposure to fluoroquinolone further increases colonization of the patient by the clonal strain, and increases the possibility of horizontal transmission from patient to patient, leading ultimately to new HAIs with cefepime resistant strains [20].

Mechanical ventilation increases the risk of acquiring nosocomial pneumonia caused by $P$. aeruginosa [21] by 3.3 times, and the same should be true for CRPA as only $62.5 \%$ of mechanical ventilation-associated pneumonia in ICU will respond to cefepime [22]. The duration of mechanical ventilation is also important since it was shown that pneumonia caused by multiresistant $P$. aeruginosa is more frequent in patients on mechanical ventilation for longer than 7 days [23]. How- 
ever, our study was the first to explicitly confirm mechanical ventilation as a risk factor for HAIs with CRPA, both carbapenem-resistant, and carbapenem-sensitive.

Traumatic injury is also an important risk factor for HAIs with $P$. aeruginosa, either indirectly (e.g. spinal injuries require bladder catheterization, which leads later on to urinary tract infection with Gram-negative bacteria including Pseudomonas [24]) or directly (e.g. thoracic injury decreases normal ventilation of lung segments and elimination of respiratory secretions). A recent systematic review of infections in patients with thoracic trauma treated using chest drains [25] was conclusive about the recommendation that all patients with thoracic injury and chest drain should receive antibiotic prophylaxis to prevent pleural empyema, which is a type of HAI. It is of great practical importance to prevent HAIs in injured patients, as it has been shown that sepsis in such patients increases the chance of mortality 6.6 times, and other infections about 1.5-1.9 times [26]. HAIs in injured patients also bear higher treatment costs, which are 2.5 times higher than in patients without HAIs.

The limitations of our study include unicentricity, which may introduce bias due to certain local practices not present in other settings and the specific conditions of the hospital where the research was conducted. A second potential limitation is selection bias, which is always a concern in case-control studies. We attempted to reduce this to a minimum by including all patients with HAIs caused by $P$. aeruginosa. The third limitation is the relatively limited scope of study variables that were taken into account. We were also restricted by our inability to perform genotyping isolates to identify clones. Despite these limitations, the data offer important information on the risk factors of HAIs caused by CRPA and provide the basis for further research involving multiple factors in order to prevent the spread of antimicrobial resistance in bacteria in hospitals and inform the introduction of prevention measures.

\section{CONCLUSION}

Our study showed that risk factors for HAIs with CRPA are primarily linked to pre-infection use of systemic antibiotics, which rapidly selects multi, extensive, or pan-resistant strains of $P$. aeruginosa, that invade tissues and cause infection. All the other risk factors that we detected (prolonged stay in hospital or ICU, other hospital infections, mechanical ventilation, and injury) lead to increased use of antibiotics, which is a common way for all these factors to induce HAI in general, including that caused by CRPA. Finally, the results of this research set the basis for the implementation of corrective measures to improve the situation we face with healthcare-associated infections. .

\section{ACKNOWLEDGMENTS}

This study was partially financed by Research grant No. 175007 given by Serbian Ministry of Education, Science and Technological Development.

\section{CONFLICT OF INTEREST}

All authors declare no conflict of interest.

\section{REFERENCES}

1. Athanasiou $\mathrm{Cl}$, Kopsini $\mathrm{A}$. A systematic review on the use of time series data in the study of antimicrobial consumption and Pseudomonas aeruginosa resistance. J Glob Antimicrob Resist. 2018;15:6973. doi: 10.1016/j.jgar.2018.06.001.

2. Boucher HW, Talbot GH, Bradley JS, Edwards JE, Gilbert D, Rice LB, et al. Bad bugs, no drugs: no ESKAPE! An update from the Infectious Diseases Society of America. Clin Infect Dis. 2009;48(1):1-12. doi: $10.1086 / 595011$.

3. Vincent JL, Rello J, Marshall J, Silva E, Anzueto A, Martin CD, et al. International study of the prevalence and outcomes of infection in intensive care units. JAMA. 2009; 302(21):2323-9. doi: 10.1001/ jama.2009.1754.

4. Nathwani D, Raman G, Sulham K, Gavaghan M, Menon V. Clinical and economic consequences of hospital-acquired resistant and multidrug-resistant Pseudomonas aeruginosa infections: a systematic review and meta-analysis. Antimicrob Resist Infect Control. 2014;3(1):32. doi: 10.1186/2047-2994-332.

5. Gupta R, Malik A, Rizvi M, Ahmed SM. Incidence of multidrug-resistant Pseudomonas spp. in ICU patients with special reference to ESBL, AMPC, MBL and biofilm production. J Glob Infect Dis. 2016;8: 25-31. doi: 10.4103/0974-777X.176142.

6. Dantas RC, Ferreira ML, Gontijo-Filho PP, Ribas RM. Pseudomonas aeruginosa bacteraemia: independent risk factors for mortality and impact of resistance on outcome. J Med Microbiol. 2014;63(Pt 12):1679-87. doi: 10.1099/jmm.0.073262-0

7. Yahav D, Paul M, Fraser A, Sarid N, Leibovici L. Ef- 
ficacy and safety of cefepime: a systematic review and meta-analysis. Lancet Infect Dis. 2007;7(5):33848. doi: 10.1016/S1473-3099(07)70109-3.

8. Akhabue E, Synnestvedt M, Weiner MG, Bilker WB, Lautenbach E. Cefepime-resistant Pseudomonas aeruginosa. Emerg Infect Dis. 2011;17(6):103743. doi: $10.3201 /$ eid/1706.100358.

9. Nasiri MI, Naqvi SB-S, Zaidi AA, Saeed R, Raza G. Comparative study on resistant pattern of clinical isolates against levofloxacin and cefepime. Pak J Pharm Sci. 2013;26(2):415-9.

10. Garner JS, Jarvis WR, Emori TG, Horan TC, Hughes JM. CDC definitions for nosocomial infections. Am J Infect Control. 1988;16:128-40.

11. Kiska DL, Gilligan PH. Pseudomonas and Burkholderia. In: Murray PR, Baron EJ, Pfaller MA et al., editors. Manual of clinical microbiology. Washington [DC]: American Society for Microbiology; 1995. p. 517-25.

12. CLSI: Clinical and Laboratory Standard Institute (CLSI), Performance standards for antimicrobial susceptibility testing. Wayne: PA, USA; 2010.

13. Magiorakos AP, Srinivasan A, Carey RB, Carmeli Y, Falagas ME, Giske CG, et al. Multidrug-resistant, extensively drug-resistant and pandrug-resistant bacteria: an international expert proposal for interim standard definitions for acquired resistance. Clin Microbiol Infect. 2012;18(3):268-81. doi: 10.1111/j.1469-0691.2011.03570.x

14. Zhao XF, Yuan M, Chen X, Liu XF, Yu DS, Li J. Drug resistance and dissemination of New Delhi metalloB-lactamase 1 positive bacteria in a patient. Zhonghua Yu Fang Yi Xue Za Zhi. 2017;51(10):890-5. doi: 10.3760/cma.j.issn.0253-9624.2017.10.005. (Article in Chinese with an abstract in English)

15. Radan M, Moniri R, Khorshidi A, Gilasi H, Norouzi $Z$, Beigi $F$, et al. Emerging Carbapenem-Resistant Pseudomonas aeruginosa Isolates Carrying blalMP Among Burn Patients in Isfahan, Iran. Arch Trauma Res. 2016;5(3):e33664. doi: 10.5812/atr.33664.

16. Zavascki AP, Barth AL, Gaspareto PB, Gonçalves ALS, Moro ALD, Fernandes JF, et al. Risk factors for nosocomial infections due to Pseudomonas aeruginosa producing metallo- $B$-lactamase in two tertiary-care teaching hospitals. J Antimicrob Chemother. 2006;58(4):882-5. doi: 10.1093/jac/dkl327.

17. Drusano GL, Bonomo RA, Bahniuk N, Bulitta JB, VanScoy B, DeFiglio H, et al. Resistance Emergence Mechanism and Mechanism of Resistance Suppression by Tobramycin for Cefepime for Pseudomonas aeruginosa. Antimicrob Agents Chemother. 2012;56(1):231-42. doi: 10.1128/AAC.05252-11.

18. Lucena A, Dalla Costa LM, Nogueira KS, Matos AP, Gales AC, Paganini MC, et al. Nosocomial infections with metallo-beta-lactamase-producing Pseudomonas aeruginosa: molecular epidemiology, risk factors, clinical features and outcomes. J Hosp Infect. 2014;87(4):234-40. doi: 10.1016/j. jhin.2014.05.007.

19. Wanis $M$, Walker SAN, Daneman N, Elligsen $M$, Palmay L, Simor A, et al. Impact of hospital length of stay on the distribution of Gram negative bacteria and likelihood of isolating a resistant organism in a Canadian burn center. Burns. 2016;42(1):10411. doi: $10.1016 /$ j.burns.2015.07.010.

20. Nouér SA, Nucci $M$, de-Oliveira MP, Pellegrino FLPC, Moreira BM. Risk factors for acquisition of multidrug-resistant Pseudomonas aeruginosa producing SPM metallo-beta-lactamase. Antimicrob Agents Chemother. 2005;49(9):3663-7. doi: 10.1128/AAC.49.9.3663-3667.2005.

21. Zhou F, Li H, Gu L, Liu M, Xue C-X, Cao B, et al. Risk factors for nosocomial infection among hospitalised severe influenza $\mathrm{A}(\mathrm{H} 1 \mathrm{~N} 1) \mathrm{pdm} 09 \mathrm{pa}-$ tients. Respir Med. 2018;134:86-91. doi: 10.1016/j. rmed.2017.11.017.

22. Xiao H, Ye X, Liu Q, Li L. Antibiotic susceptibility and genotype patterns of Pseudomonas aeruginosa from mechanical ventilation-associated pneumonia in intensive care units. Biomed Rep. 2013;1(4):58993. doi: $10.3892 / \mathrm{br} .2013 .94$.

23. Rello J, Allegri C, Rodriguez A, Vidaur L, Sirgo G, Gomez F, et al. Risk Factors for Ventilator-associated Pneumonia by Pseudomonas aeruginosa in Presence of Recent Antibiotic Exposure. Anesthes. 2006;105(4):709-14.

24. Montgomerie JZ. Infections in patients with spinal cord injuries. Clin Infect Dis. 1997;25(6):128590.

25. Bosman A, de Jong MB, Debeij J, van den Broek PJ, Schipper IB. Systematic review and meta-analysis of antibiotic prophylaxis to prevent infections from chest drains in blunt and penetrating thoracic injuries. $\mathrm{Br}$ J Surg. 2012;99(4):506-13. doi: $10.1002 /$ bjs. 7744 .

26. Glance LG, Stone PW, Mukamel DB, Dick AW. Increases in mortality, length of stay, and cost associated with hospital-acquired infections in trauma patients. Arch Surg. 2011;146(7):794-801. doi: 10.1001/archsurg.2011.41. 


\title{
Faktori rizika za nastanak bolničkih infekcija uzrokovanih Pseudomonas aeruginosa rezistentnim na cefepim
}

\author{
Marko M. Folić1,2, Zorana M. Djordjević́3, Dragan R. Milovanovićc, \\ Dejana T. Ružić Zečević, ${ }^{1,2}$, Nikola V. Rosić ${ }^{1}$, Slobodan M. Jankovićc ${ }^{1,2}$ \\ ${ }^{1}$ Klinički centar Kragujevac, Služba za kliničku farmakologiju, Kragujevac, Srbija \\ ${ }^{2}$ Univerzitet u Kragujevcu, Fakultet medicinskih nauka, Kragujevac, Srbija \\ ${ }^{3}$ Klinički centar Kragujevac, Odsek za kontrolu bolničkih infekcija, Kragujevac, Srbija
}

\section{KRATAK SADRŽAJ}

Uvod: Porast rezistencije Pseudomonas aeruginosa na cefepim zahteva bolje razumevanje bolničke epidemiologije u cilju zadržavanja ovog medikamenta kao adekvatne terapijske opcije.

Cilj: Cilj ove studije bazirao se na identifikaciji faktora rizika udruženih sa razvojem bolničkih infekcija uzrokovanih $P$. aeruginosa rezistentnim na cefepim, kako kod svih bolesnika sa infekcijama ovog tipa, tako i kod podgrupe pacijenata sa letalnim ishodom i podgrupe onih koji su razvili infekciju uzrokovanu $P$. aeruginosa otpornim na karbapeneme.

Metod: Ova akademska studija je dizajnirana kao kombinacija tri „ugnježdene“ studije tipa slučaj/kontrola u okviru kohorte pacijenata sa bolničkim infekcijama uzrokovanim $P$. aeruginosa bez obzira na lokaciju, prospektivno praćene u periodu od januara 2009. do decembra 2017. godine u ustanovi tercijarnog nivoa zdravstvene zaštite. Uticaj faktora rizika na specifične ishode procenjivan je na osnovu prilagođenih odnosa šansi izračunatih iz multivarijantnih logističkih regresija.

Rezultati: Naše istraživanje je pokazalo da su nezavisni faktori rizika za nastanak infekcija uzrokovanih $P$. aeruginosa rezistentnim na cefepim prisustvo dodatne intrahospitalne infekcije kod pacijenta $(p=0,001)$, hospitalizacija u jednici intenzivne nege duža od jednog meseca ( $p=0,007)$, duže vreme hospitalizacije pre nastanka infekcije $(p=0,004)$, dugotrajna primena mehaničke ventilacije $(p=0,036)$, primena antibiotika pre razvoja infekcije $(p<0,001)$ i prethodna upotreba fluorohinolona $(p=0,030)$, dok su traumatske povrede $(p=0,020)$, postojanje dodatne bolničke infekcije $(p=0,001)$ i dugotrajna primena antibiotika pre razvoja bolničke infekcije $(p=0,034)$ povezane sa smrtnošću. Nezavisni faktori rizika za razvoj intrahospitalnih infekcija uzrokovanih $P$. aeruginosa rezistentnim na cefepim koji je ujedno otporan i na karbapeneme su postojanje dodatne intrahospitalne infekcije $(p=0,011)$, prelazak pacijenta iz druge organizacione jedinice $(p=0,023)$, duži period boravka u bolnici $(p=0,006)$ i jedinici intenzivne nege $(p=0,004)$ i primena antibiotika pre razvoja infekcije $(p<0,001)$.

Zaključak: Faktori rizika za razvoj bolničkih infekcija uzrokovanih $P$. aeruginosa rezistentnim na cefepim primarno su povezani sa primenom sistemskih antibiotika pre razvoja infekcije, što dovodi do selekcije multi, ekstenzivnih ili pan-rezistentnih sojeva $P$. aeruginosa koji napadaju tkiva i izazivaju infekciju.

Ključne reči: Pseudomonas aeruginosa, cefepim, rezistencija na antibiotike, faktori rizika 